Bull. Austral. Math. Soc.

$16 \mathrm{U} 99,16 \mathrm{~s} 50$

VoL. 74 (2006) [279-288]

\title{
COMMUTATOR RINGS
}

\section{ZACHARY MESYAN}

A ring is called a commutator ring if every element is a sum of additive commutators. In this note we give examples of such rings. In particular, we show that given any ring $R$, a right $R$-module $N$, and a nonempty set $\Omega, \operatorname{End}_{R}\left(\bigoplus_{\Omega} N\right)$ and $\operatorname{End}_{R}\left(\Pi_{\Omega} N\right)$ are commutator rings if and only if either $\Omega$ is infinite or $\operatorname{End}_{R}(N)$ is itself a commutator ring. We also prove that over any ring, a matrix having trace zero can be expressed as a sum of two commutators.

\section{INTRODUCTION}

In 1956 Irving Kaplansky proposed twelve problems in ring theory (see [5]). One of these was whether there is a division ring in which every element is a sum of additive commutators. This was answered in the affirmative two years later by Harris in [4]. However, it seems that to this day not much is known about rings in which every element is a sum of commutators, which we shall call commutator rings.

The purpose of the first half of this note is to collect examples of such rings. For instance, we shall show that given any ring $R$, a right $R$-module $N$, and a nonempty set $\Omega, \operatorname{End}_{R}\left(\bigoplus_{\Omega} N\right)$ and $\operatorname{End}_{R}\left(\prod_{\Omega} N\right)$ are commutator rings if and only if either $\Omega$ is infinite or $\operatorname{End}_{R}(N)$ is itself a commutator ring. We shall also note that if $R$ is a nonzero commutative ring and $n$ is a positive integer, then the Weyl algebra $A_{n}(R)$ is a commutator ring if and only if $R$ is a $Q$-algebra.

Along the way, we shall give an alternate characterisation of right $R$-modules $M$ that can be written in the form $\bigoplus_{i \in \Omega} N_{i}$, where $\Omega$ is an infinite set, and the right $R$-modules $N_{i}$ are all isomorphic. Specifically, $M$ is of this form if and only if there exist $x, z \in \operatorname{End}_{R}(M)$ such that $z x=1$ and $\bigcup_{i=1}^{\infty} \operatorname{ker}\left(z^{i}\right)=M$.

The last section of this note is devoted to commutators in matrix rings. In [1] Albert and Muckenhoupt showed that if $F$ is a field and $n$ is a positive integer, then every matrix in $\mathbf{M}_{n}(F)$ having trace zero can be expressed as a commutator in that ring. (This was first proved for fields of characteristic zero by Shoda in [11].) The question of whether

\section{Received 8th May, 2006}

The author is grateful to George Bergman for his numerous comments and suggestions on earlier drafts of this note, and to Lance Small for his comments and references to related literature.

Copyright Clearance Centre, Inc. Serial-fee code: 0004-9727/06 \$A2.00+0.00. 
this result could be generalised to arbitrary rings remained open for a number of years, until Rosset and Rosset showed in [10] that it could not. (An example demonstrating this will also be given below.) However, we shall prove that every matrix having trace zero can be expressed as a sum of two commutators, generalising a result of Rosset in [8] (unpublished) for matrices over commutative rings. As a corollary, we also obtain a generalisation to arbitrary rings of the result in [4] that a matrix over a division ring is a sum of commutators if and only if its trace is a sum of commutators. On a related note, in [2] Amitsur and Rowen showed that if $R$ is a finite-dimensional central simple algebra over a field $F$, then every element $r \in R$ such that $r \otimes 1$ has trace zero in $R \otimes_{F} \bar{F} \cong \mathbf{M}_{n}(\bar{F})$ is a sum of two commutators, where $\bar{F}$ is the algebraic closure of $F$. (See also [9].)

\section{Definitions AND EXAMPLES}

Given a unital associative ring $R$ and two elements $x, y \in R$, let $[x, y]$ denote the commutator $x y-y x$. We note that $[x, y]$ is additive in either variable and

$$
[x, y z]=[x, y] z+y[x, z],[z x, y]=[z, y] x+z[x, y]
$$

are satisfied by all $x, y, z \in R$ (that is, $[x,-]$ and $[-, y]$ are derivations on $R$ ). Let $[R, R]$ denote the additive subgroup of $R$ generated by the commutators in $R$, and given an element $x \in R$, let $[x, R]=\{[x, y]: y \in R\}$. If $n$ is a positive integer, we shall denote the set of sums of $n$ commutators in elements of $R$ by $[R, R]_{n}$. For convenience, we define $[R, R]_{0}=\{0\}$. Finally, right module endomorphisms will be written on the left of their arguments.

Definition 1: A ring $R$ is called a commutator ring if $R=[R, R]$.

In [4] and [6] examples of commutator division rings are constructed. Also, it is easy to see that finite direct products and homomorphic images of commutator rings are commutator rings.

PROPOSITION 2. If $R \subseteq S$ are rings such that $R$ is a commutator ring and $S$ is generated over $R$ by elements centralising $R$, then $S$ is also a commutator ring.

Proof: Given an element $a \in S$, we can write $a=\sum_{i=1}^{m} r_{i} s_{i}$, where $r_{i} \in R$, the $s_{i} \in S$ centralise $R$, and $m$ is some positive integer. Since $R$ is a commutator ring, for each $r_{i}$ there are elements $y_{i j}, x_{i j} \in R$ and a positive integer $m_{i}$ such that $r_{i}=\sum_{j=1}^{m_{i}}\left[x_{i j}, y_{i j}\right]$. Then

$$
a=\sum_{i=1}^{m} r_{i} s_{i}=\sum_{i=1}^{m}\left(\sum_{j=1}^{m_{i}}\left[x_{i j}, y_{i j}\right]\right) s_{i}=\sum_{i=1}^{m}\left(\sum_{j=1}^{m_{i}}\left[x_{i j}, y_{i j} s_{i}\right]\right)
$$

This proposition implies that, for example, matrix rings, group rings, and polynomial rings over commutator rings are commutator rings. Furthermore, given a commutative 
ring $K$ and two $K$-algebras $R$ and $S$, such that $R$ is a commutator ring, $R \otimes_{K} S$ is again a commutator ring.

Given a ring $R$, a set of variables $X$, and a set of relations $Y$, we shall denote the $R$-ring presented by $R$-centralising generators $X$ and relations $Y$ by $R\langle X: Y\rangle$.

Definition 3: Let $R$ be a ring. Then $A_{1}(R)=R\langle x, y:[x, y]=1\rangle$ is called the (first) Weyl algebra over $R$. Higher Weyl algebras are defined inductively by $A_{n}(R)=A_{1}\left(A_{n-1}(R)\right)$. More generally, given a set $I$, we shall denote the ring $R\left\langle\left\{x_{i}\right\}_{i \in I},\left\{y_{i}\right\}_{i \in I}:\left[x_{i}, x_{j}\right]=\left[y_{i}, y_{j}\right]=\left[x_{i}, y_{j}\right]=0\right.$ for $i \neq j$, and $\left.\left[x_{i}, y_{i}\right]=1\right\rangle$ by $A_{I}(R)$.

Propos I T ION 4. For any ring $R$ and any infinite set $I, A_{I}(R)=\left[A_{I}(R), A_{I}(R)\right]_{1}$. In particular, any ring can be embedded in a commutator ring.

Proof: Let $s \in A_{I}(R)$ be any element. When written as a polynomial in $\left\{x_{i}\right\}_{i \in I}$ and $\left\{y_{i}\right\}_{i \in I}, s$ contains only finitely many variables $y_{i}$, so there exists some $n \in I$ such that $y_{n}$ does not occur in $s$. Then $\left[x_{n}, s\right]=0$, and hence

$$
s=1 \cdot s=\left[x_{n}, y_{n}\right] s=\left[x_{n}, y_{n} s\right]-y_{n}\left[x_{n}, s\right]=\left[x_{n}, y_{n} s\right] .
$$

Harris used this construction in [4] to produce a commutator division ring. Specifically, he took $R$ to be a field and then used essentially the method above to show that the division ring of fractions of $A_{I}(R)$ is a commutator ring.

Before discussing the case when $I$ is a finite set, we record two well known facts that will be useful.

LEMMA 5. Let $R$ be any ring and $n$ a positive integer. If $A, B \in \mathbf{M}_{n}(R)$, then trace $([A, B]) \in[R, R]_{n^{2}}$. Conversely, given any $r \in[R, R]_{n^{2}}$, there exist matrices $A, B \in \mathbf{M}_{n}(R)$ such that trace $([A, B])=r$.

Proof: Let $A, B \in \mathrm{M}_{n}(R)$, and write $A=\left(a_{i j}\right)$ and $B=\left(b_{i j}\right)$. Then

$$
\operatorname{trace}(A B-B A)=\sum_{i=1}^{n} \sum_{k=1}^{n} a_{i k} b_{k i}-\sum_{i=1}^{n} \sum_{k=1}^{n} b_{i k} a_{k i} .
$$

Now, for all $i$ and $j$ with $1 \leqslant i, j \leqslant n, a_{i j} b_{j i}$ appears exactly once in the first term of this expression, and $b_{j i} a_{i j}$ appears exactly once in the second term. Hence,

$$
\operatorname{trace}(A B-B A)=\sum_{i=1}^{n} \sum_{j=1}^{n}\left[a_{i j}, b_{j i}\right] .
$$

For the second statement, given $r \in[R, R]_{n^{2}}$, we can write $r=\sum_{i=1}^{n} \sum_{j=1}^{n}\left[a_{i j}, b_{j i}\right]$ for some $a_{i j}, b_{j i} \in R$, after relabeling. Setting $A=\left(a_{i j}\right)$ and $B=\left(b_{i j}\right)$, we have trace $([A, B])=r$, by the previous paragraph. 
Proposition 6. Let $F$ be a field and $R$ a finite-dimensional $F$-algebra. Then $R \neq[R, R]$.

Proof: Suppose that $R$ is an $F$-algebra such that $R=[R, R]$. Let $K$ be the algebraic closure of $F$ and $R_{K}=R \otimes_{F} K$ the scalar extension of $R$ to $K$. Then $R_{K}$ $=\left[R_{K}, R_{K}\right]$, by Proposition 2 . Since the property of being a commutator ring is preserved by homomorphic images, it will suffice to show that some homomorphic image of $R_{K}$ is not a commutator ring; so without loss of generality we may assume that $R_{K}$ is a simple $K$-algebra. Then, as a finite-dimensional simple algebra over an algebraically closed field, $R_{K}$ is a full matrix ring. Hence, $\left[R_{K}, R_{K}\right]$ lies in the kernel of the trace, by Lemma 5 , contradicting $R_{K}=\left[R_{K}, R_{K}\right]$.

From this it follows that, for instance, no PI ring can be a commutator ring, since every PI ring has an image that is finite-dimensional over a field.

It is well known that for any Q-algebra $R$, the $n$th Weyl algebra $A_{n}(R)$ satisfies $A_{n}(R)=\left[r, A_{n}(R)\right]$ for some $r \in A_{n}(R)$ (for example, see [3]). Actually, it is not hard to show that writing

$$
A_{n}(R)=A_{1}\left(A_{n-1}(R)\right)=A_{n-1}(R)\langle x, y:[x, y]=1\rangle,
$$

we can take $r=a x+b y+c$ for any $a, b, c$ in the centre $C$ of $R$, such that $a C+b C=C$. Combining this with another fact about Weyl algebras, we obtain the following statement.

PROPOS I TION 7. Let $R$ be a nonzero commutative ring and $n$ a positive integer. Then the Weyl algebra $A_{n}(R)$ is a commutator ring if and only if $R$ is a $Q$-algebra.

Proof: If $R$ is not a $\mathbb{Q}$-algebra, then we may assume that $R$ is a $\mathbb{Z} / p \mathbb{Z}$-algebra for some prime $p$, after passing to a quotient. By a theorem of Revoy in [7], for such a ring $R, A_{n}(R)$ is an Azumaya algebra and hence has a quotient that is a finite-dimensional algebra over a field. Therefore, by Proposition $6, A_{n}(R)$ cannot be a commutator ring.

We can also prove a more general statement.

Propos ITION 8. Let $n$ be a positive integer, $p$ a prime number, and $R$ a $\mathbb{Z} / p \mathbb{Z}$ algebra. If $R \neq[R, R]$, then $A_{n}(R) \neq\left[A_{n}(R), A_{n}(R)\right]$.

Proof: By induction on $n$, it suffices to prove the proposition for

$$
A_{1}(R)=R\langle x, y:[x, y]=1\rangle
$$

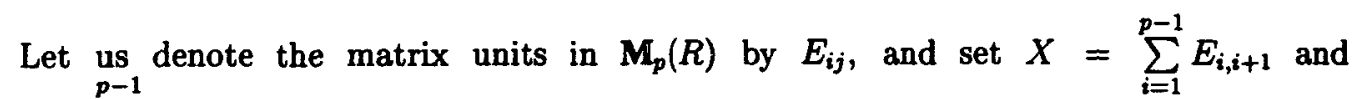
$Y=\sum_{i=1}^{p-1} i E_{i+1, i}$. Then

$$
X Y-Y X=\sum_{i=1}^{p-1} i E_{i, i}-\sum_{i=1}^{p-1} i E_{i+1, i+1}=\sum_{i=1}^{p} i E_{i, i}-\sum_{i=1}^{p}(i-1) E_{i, i}=1 .
$$


Hence $x \mapsto X$ and $y \mapsto Y$ induces a ring homomorphism from $A_{1}(R)$ to $\mathrm{M}_{p}(R)$. Now, since $i^{p-1} \equiv 1(\bmod p)$ for $i \in\{1,2, \ldots, p-1\}$, we have

$$
\operatorname{trace}\left((X Y)^{p-1}\right)=\operatorname{trace}\left(\sum_{i=1}^{p-1} i^{p-1} E_{i, i}\right)=\sum_{i=1}^{p-1} i^{p-1}=p-1 .
$$

Thus, if $r \in R \backslash[R, R]$, then trace $\left(-r(X Y)^{p-1}\right)=r \in R$, and so, by Lemma $5,-r(X Y)^{p-1}$ is not a sum of commutators in $M_{p}(R)$. Consequently, $-r(x y)^{p-1}$ is not a sum of commutators in $A_{1}(R)$.

\section{ENDOMORPHISM RINGS}

We begin with a general result about commutators in matrix rings and, with its help, provide another way of constructing commutator rings.

LEMMA 9. Let $R$ be a ring and $r \in R$ any element. Suppose that $e \in R$ is an idempotent such that ere $\in[e R e, e R e]_{m_{1}}$ and $f r f \in[f R f, f R f]_{m_{2}}$, where $f=1-e$ and $m_{1}, m_{2} \in \mathbb{N}$. Then $r \in[R, R]_{m_{2}+1}$, where $m=\max \left(m_{1}, m_{2}\right)$.

Proof: First, we note that for any

$$
x, y, z, w \in R,[e x e, e y e]+[f z f, f w f]=[e x e+f z f, e y e+f w f]
$$

Hence, if ere $\in[e R e, e R e]_{m}$ and $f r f \in[f R f, f R f]_{m}$, then ere $+f r f \in[R, R]_{m}$.

Now, write $r=e r e+e r f+f r e+f r f$. Then erf $=(-e r f) e-e(-e r f)$ and $f r e=(f r e) e-e(f r e)$. Hence, $r=[f r e-e r f, e]+e r e+f r f \in[R, R]_{m+1}$.

Proposition 10. Let $R$ be a ring, $r \in R$ any element, and $m_{1}, m_{2}, \ldots, m_{n}$ $\in \mathbb{N}$. Suppose that $e_{1}, e_{2}, \ldots, e_{n} \in R$ are orthogonal idempotents such that $1=e_{1}+e_{2}$ $+\cdots+e_{n}$ and $e_{i} r e_{i} \in\left[e_{i} R e_{i}, e_{i} R e_{i}\right]_{m_{i}}$ for $i=1,2, \ldots, n$ (where each $e_{i} R e_{i}$ is viewed as a ring with identity $\left.e_{i}\right)$. Then $r \in[R, R]_{m+n-1}$, where $m=\max \left(m_{1}, m_{2}, \ldots, m_{n}\right)$.

Proof: We shall proceed by induction on $n$. The statement is a tautology when $n=1$. If $n>1$, let $f=e_{1}+e_{2}+\cdots+e_{n-1}$. Then $f=f^{2}$ and $f=1-e_{n}$. Also, for

$$
i \in\{1,2, \ldots, n-1\}, e_{i}(f r f) e_{i}=e_{i} r e_{i} \in\left[e_{i} R e_{i}, e_{i} R e_{i}\right]_{m_{i}}=\left[e_{i}(f R f) e_{i}, e_{i}(f R f) e_{i}\right]_{m_{i}},
$$

so, by the inductive hypothesis, $f r f \in[f R f, f R f]_{\max \left(m_{1}, m_{2}, \ldots, m_{n-1}\right)+n-2}$. But, by assumption, $e_{n} r e_{n} \in\left[e_{n} R e_{n}, e_{n} R e_{n}\right]_{m_{n}}$. Hence, $r \in[R, R]_{m+n-1}$, by the preceding lemma and the fact that $\max \left(\max \left(m_{1}, m_{2}, \ldots, m_{n-1}\right)+n-2, m_{n}\right) \leqslant \max \left(m_{1}, m_{2}, \ldots, m_{n}\right)+n-2$.

Corollary 11. Let $R$ be a ring and $M=M_{1} \oplus M_{2} \oplus \cdots \oplus M_{n}$ be right $R$ modules. If each $\operatorname{End}_{R}\left(M_{i}\right)$ is a commutator ring, then so is $\operatorname{End}_{R}(M)$. Also, if for each $i$ there is some positive integer $m_{i}$ such that $\operatorname{End}_{R}\left(M_{i}\right)=\left[\operatorname{End}_{R}\left(M_{i}\right), \operatorname{End}_{R}\left(M_{i}\right)\right]_{m_{i}}$, then $\operatorname{End}_{R}(M)=\left[\operatorname{End}_{R}(M), \operatorname{End}_{R}(M)\right]_{m+n-1}$, where $m=\max \left(m_{1}, m_{2}, \ldots, m_{n}\right)$. 
Let us now turn to endomorphism rings of infinite direct sums and products of copies of a fixed module.

Proposition 12. Let $R$ be a ring, $N$ a right $R$-module, $\Omega$ an infinite set, and $M=\bigoplus_{\Omega} N$ or $M=\prod_{\Omega} N$. Then $\operatorname{End}_{R}(M)=\left[x, \operatorname{End}_{R}(M)\right]$ for some $x \in \operatorname{End}_{R}(M)$. If $\Omega=\mathbb{N}$, then $x$ can be taken to be the right shift operator.

Proof: Since $\bigoplus_{\Omega} N \cong \bigoplus_{i=0}^{\infty}\left(\bigoplus_{\Omega} N\right)$ and $\Pi_{\Omega} N \cong \prod_{i=0}^{\infty}\left(\prod_{\Omega} N\right)$, it suffices to prove the proposition in the case $\Omega \stackrel{i=\mathbb{N}}{=}$. Let $x$ be the right shift operator and $z$ the left shift operator. Now, consider any endomorphism $f \in \operatorname{End}_{R}(M)$, and set $y=-\sum_{i=0}^{\infty} x^{i} f z^{i+1}$. Assuming this summation converges and using the relation $z x=1$, we have

$$
x y-y x=-\sum_{i=0}^{\infty} x^{i+1} f z^{i+1}+\sum_{i=0}^{\infty} x^{i} f z^{i+1} x=-\sum_{i=1}^{\infty} x^{i} f z^{i}+\sum_{i=0}^{\infty} x^{i} f z^{i}=f .
$$

It remains to prove convergence of the sum defining $y$ in the function topology on End $_{R}(N)$. (In the case $M=\bigoplus_{\Omega} N$, it is the topology based on regarding $M$ as a discrete module, while in the case $M=\prod_{\Omega} N$, it is the topology constructed using the product topology on $M$, arising from the discrete topologies on the factors.)

If $M=\bigoplus_{\Omega} N$, then $\bigcup_{i=1}^{\infty} \operatorname{ker}\left(z^{i}\right)=M$. Hence, every element of $M$ is annihilated by almost all summands of $-\sum_{i=0}^{\infty} x^{i} f z^{i+1}$. If $M=\prod_{\Omega} N$, then given any positive integer $i$ and any $m \in M, x^{j} f z^{j+1}(m)$ has a nonzero component in the copy of $N$ indexed by $i$ for only finitely many values of $j\left(\right.$ namely, for $j \leqslant i$ ). In either case, $-\sum_{i=0}^{\infty} x^{i} f z^{i+1}$ converges
in the appropriate topology on $\operatorname{End}_{R}(M)$.

Using similar methods, it can be shown that given any ring $R$, the ring of infinite matrices over $R$ that are both row-finite and column-finite is also a commutator ring.

THEOREM 13. Let $R$ be a ring, $N$ a right $R$-module, $\Omega$ a nonempty set, and $M=\bigoplus_{\Omega} N$ or $\prod_{\Omega} N$. Then $\operatorname{End}_{R}(M)$ is a commutator ring if and only if either $\Omega$ is infinite or $\operatorname{End}_{R}(N)$ is a commutator ring.

Proof: Suppose that $\operatorname{End}_{R}(M)$ is a commutator ring and $\Omega$ is finite. Then $\operatorname{End}_{R}(M) \cong M_{n}\left(\operatorname{End}_{R}(N)\right)$ for some positive integer $n$. Hence, $\operatorname{End}_{R}(N)$ is a commutator ring, by Lemma 5 . The converse follows from the previous proposition if $\Omega$ is infinite, and from Proposition 2 if $\Omega$ is finite.

Incidentally, in the proof of Proposition 12, the only fact about $M=\bigoplus_{i=0}^{\infty} N$ that we used was that for such a module there are endomorphisms $x, z \in \operatorname{End}_{R}(M)$ such that $z x=1$ and $\bigcup_{i=1}^{\infty} \operatorname{ker}\left(z^{i}\right)=M$. This condition actually characterises modules that are infinite direct sums of copies of a module. 
Proposition 14. Let $R$ be a ring and $M$ a right $R$-module. The following are equivalent.

(1) $\quad M=\bigoplus_{i \in \Omega} N_{i}$ for some infinite set $\Omega$ and some right $R$-modules $N_{i}$, such that $N_{i} \cong N_{j}$ for all $i, j \in \Omega$.

(1') $\quad M=\bigoplus_{i \in \Omega} N_{i}$ for some countably infinite set $\Omega$ and some right $R$-modules $N_{i}$, such that $N_{i} \cong N_{j}$ for all $i, j \in \Omega$.

(2) There exist $x, z \in \operatorname{End}_{R}(M)$ such that $z x=1$ and $\bigcup_{i=1}^{\infty} \operatorname{ker}\left(z^{i}\right)=M$.

Proof: The equivalence of $(1)$ and $\left(1^{\prime}\right)$ is clear. To deduce (2) from $\left(1^{\prime}\right)$, we may assume that $M=\bigoplus_{i=0}^{\infty} N_{i}$, after well ordering $\Omega$. Then $x$ can be taken to be the right shift operator and $z$ the left shift operator. To show that (2) implies (1'), let $N_{i}=x^{i} \operatorname{ker}(z)$ for each $i \in \mathbb{N}$. Taking $i \geqslant 1$ and $a \in \operatorname{ker}(z)$, we have $z x^{i}(a)=x^{i-1}(a)$. Hence, left multiplication by $z$ gives a right $R$-module homomorphism $N_{i} \rightarrow N_{i-1}$, which is clearly surjective. This homomorphism is also injective, since $N_{i}=x\left(x^{i-1} \operatorname{ker}(z)\right)$ and $z x=1$. By induction, $N_{i} \cong N_{j}$ for all $i, j \in \mathbb{N}$.

Next, let us show that $\sum_{i=0}^{\infty} N_{i}$ is direct. Suppose that $a_{0}+a_{1}+\cdots+a_{n}=0$, where each $a_{i} \in N_{i}, n \geqslant 1$, and $a_{n} \neq 0$. For each $i \in\{0,1, \ldots, n\}$, write $a_{i}=x^{i}\left(b_{i}\right)$, where $b_{i} \in \operatorname{ker}(z)$. Then

$$
0=z^{n}\left(a_{0}+a_{1}+\cdots+a_{n}\right)=z^{n}\left(b_{0}\right)+z^{n-1}\left(b_{1}\right)+\cdots+z\left(b_{n-1}\right)+b_{n}=b_{n} .
$$

Hence, $a_{n}=0 ;$ a contradiction.

Finally, we show that given $a \in M$, we have $a \in \bigoplus_{i=0}^{\infty} N_{i}$. By $(2), a \in \operatorname{ker}\left(z^{n}\right)$ for some positive integer $n$. If $n=1$, then $a \in N_{0}$, so there is nothing to prove. Otherwise, $z a \in \operatorname{ker}\left(z^{n-1}\right)$, and we may assume inductively that $z a=x^{0}\left(b_{0}\right)+x^{1}\left(b_{1}\right)+\cdots+x^{n-1}\left(b_{n-1}\right)$ for some $b_{0}, b_{1}, \ldots, b_{n-1} \in \operatorname{ker}(z)$. Then $a=(a-x z a)+x z a$, where $a-x z a \in \operatorname{ker}(z)=N_{0}$, and $x z a \in \bigoplus_{i=1}^{n} N_{i}$.

\section{Traceless matrices}

We now prove our main result about commutators in matrix rings. This proof uses the same fundamental idea as the one for Proposition 12.

THEOREM 15. Let $R$ be a ring and $n$ a positive integer. Then there exist matrices $X, Y \in \mathbf{M}_{n}(R)$ such that for all $A \in \mathbf{M}_{n}(R)$ having trace $0, A \in\left[X, \mathbf{M}_{n}(R)\right]+\left[Y, \mathbf{M}_{n}(R)\right]$. Specifically, writing $E_{i j}$ for the matrix units, one can take $X=\sum_{i=1}^{n-1} E_{i+1, i}$ and $Y=E_{n n}$.

Proof: Write $A=\left(a_{i j}\right)$, and set $X=\sum_{i=1}^{n-1} E_{i+1, i}, Z=\sum_{i=1}^{n-1} E_{i, i+1}$. Then

$$
Z X=E_{11}+E_{22}+\cdots+E_{n-1, n-1}=I-E_{n n} .
$$


Hence,

(1) $Z X=I-E_{n n}$.

Also, for $l \in\{0,1, \ldots, n-1\}$,

$$
E_{n n} X^{l} A Z^{l} E_{n n}=E_{n n}\left(\sum_{i=1}^{n-l} E_{i+l, i}\right) A\left(\sum_{i=1}^{n-l} E_{i, i+l}\right) E_{n n}=E_{n, n-l} A E_{n-l, n}=a_{n-l, n-l} E_{n n} .
$$

Thus,

(2) $E_{n n} X^{l} A Z^{l} E_{n n}=a_{n-l, n-l} E_{n n}$.

Now, let

$$
C=A+X A Z+\cdots+X^{n-1} A Z^{n-1} .
$$

Then

$$
[C Z, X]=C Z X-X C Z=C\left(I-E_{n n}\right)-X C Z \text {, }
$$

by (1). Also, $C-X C Z=A$, since $X^{n}=0$. Hence, $[C Z, X]=A-C E_{n n}$. We note that $C E_{n n}$ is an $R$-linear combination of $E_{1 n}, E_{2 n}, \ldots, E_{n-1, n}$, since

$$
E_{n n} C E_{n n}=\left(a_{n n}+a_{n-1, n-1}+\cdots+a_{11}\right) E_{n n}=0 \text {, }
$$

by (2) and the hypothesis that $\operatorname{trace}(A)=0$.

Setting $Y=E_{n n}$, we have for each $i \in\{1,2, \ldots, n-1\}, E_{i n}=E_{i n} Y-Y E_{i n}$. Hence $C E_{n n}=\left[C E_{n n}, Y\right]$, and therefore $A=[C Z, X]+\left[C E_{n n}, Y\right]$.

Corollary 16. Let $R$ be a ring, $n$ a positive integer, $m \in \mathbb{N}$, and $A \in \mathbb{M}_{n}(R)$. If trace $(A) \in[R, R]_{m}$, then $A \in\left[\mathbf{M}_{n}(R), \mathbf{M}_{n}(R)\right]_{\left\lceil m / n^{2}\right\rceil+2}$, where $\left\lceil m / n^{2}\right\rceil$ denotes the least integer $\geqslant m / n^{2}$.

Proof: Let $r=\operatorname{trace}(A)$. By Lemma 5, there is a matrix $B \in\left[\mathbf{M}_{n}(R), \mathbf{M}_{n}(R)\right]_{\left[m / n^{2}\right]}$ such that $\operatorname{trace}(B)=r$. By the previous theorem, $A-B$ is a sum of two commutators. Hence, $A \in\left[\mathbf{M}_{n}(R), \mathbf{M}_{n}(R)\right]_{\left\{m / n^{2}\right\}+2}$.

Corollary 17. Let $R$ be a ring, $n$ a positive integer, and $A \in \mathbf{M}_{n}(R)$ a matrix. Then $A \in\left[\mathbf{M}_{n}(R), \mathbf{M}_{n}(R)\right]$ if and only trace $(A) \in[R, R]$.

Proof: The forward implication was proved in Lemma 5, while the converse follows from the previous corollary.

This is a generalisation of the result in [4] that given a division ring $D$, a matrix $A \in \mathbf{M}_{n}(D)$ is a sum of commutators if and only if its trace is a sum of commutators in $D$. Actually, Corollary 17 can also be deduced rather quickly from Proposition 10 by writing $A=B+C$, where $B \in \mathbf{M}_{n}(R)$ has zeros on the main diagonal, $C \in \mathbf{M}_{n}(R)$ has zeros everywhere off the main diagonal, and $\operatorname{trace}(C) \in[R, R]$. Then $B \in\left[\mathbf{M}_{n}(R), \mathbf{M}_{n}(R)\right]$, by the proposition, and $C$ can be written as a sum of commutators and matrices of the form $x E_{i i}-x E_{n n}=\left(x E_{i n}\right) E_{n i}-E_{n i}\left(x E_{i n}\right)$. 
Let us now give an example of a matrix that has trace 0 but is not a commutator, showing that in general the number of commutators in Theorem 15 cannot be decreased to one. A similar example appears in [10], however, our proof is considerably shorter. We shall require the following lemma in the process.

LEMMA 18. Let $F$ be a field and $A, B \in \mathbf{M}_{2}(F)$ matrices such that $[A, B]=0$. Then $\left\{[A, C]+[B, D]: C, D \in \mathbf{M}_{2}(F)\right\}$ is a subspace of the $F$-vector space $\mathbf{M}_{2}(F)$ of dimension at most 2 .

Proof: The result is clear if $A$ and $B$ are both central in $\mathbf{M}_{2}(F)$, so we may assume that $A$ is not central. For all $C \in \mathbb{M}_{2}(F)$ let $f(C)=[A, C]$ and $g(C)=[B, C]$. Then $f$ and $g$ are $F$-vector space endomorphisms of $\mathbf{M}_{2}(F)$. Let $S \subseteq \mathbf{M}_{2}(F)$ be the subspace spanned by the images of $f$ and $g$. We shall show that $S$ is at most 2-dimensional.

For all $a, b, c \in F$ we have $f(a A+b B+c I)=0=g(a A+b B+c I)$, since $[A, B]=0$. If $B$ is not in the span of $I$ and $A$, then $\operatorname{dim}_{F}(\operatorname{ker}(f)) \geqslant 3$ and $\operatorname{dim}_{F}(\operatorname{ker}(g)) \geqslant 3$. So im(f) and $\operatorname{im}(g)$ are each at most 1-dimensional, and hence $S$ can be at most 2-dimensional. Therefore, we may assume that $B=a A+b I$ for some $a, b \in F$. In this case, $S=\operatorname{im}(f)$. But, $I, A \in \operatorname{ker}(f)$, and $A$ is not in the span of $I$, so $\operatorname{im}(f)$ is at most 2-dimensional, as desired.

PRoposition 19. Let $F$ be a field and $R=F\left[x_{11}, x_{12}, x_{21}\right] /\left(x_{11}, x_{12}, x_{21}\right)^{2}$. Then the matrix

$$
X=\left(\begin{array}{cc}
\bar{x}_{11} & \bar{x}_{12} \\
\bar{x}_{21} & -\bar{x}_{11}
\end{array}\right) \in \mathbf{M}_{2}(R)
$$

has trace 0 but is not a commutator in $\mathbf{M}_{2}(R)$.

Proof: Suppose that $X=[A, B]$ for some $A, B \in R$. Viewing $X, A$, and $B$ as polynomials in $\bar{x}_{11}, \bar{x}_{12}$, and $\bar{x}_{21}$, let $A_{0}, B_{0} \in \mathbf{M}_{2}(F)$ denote the degree-0 terms of $A$ and $B$, respectively. Then the equation $X=[A, B]$ tells us that $\left[A_{0}, B_{0}\right]=0$ and that the matrices of the form $\left[A_{0}, C\right]-\left[B_{0}, D\right]\left(C, D \in \mathbf{M}_{2}(F)\right)$ span a 3-dimensional subspace of $\mathbf{M}_{2}(F)$, namely the subspace of all trace-0 matrices. (For if we denote the coefficients of $\bar{x}_{i j}$ in $A$ and $B$ by $A_{i j}$ and $B_{i j}$, respectively, then the coefficient of $\bar{x}_{i j}$ in $X$ is $\left[A_{0}, B_{i j}\right]+\left[A_{i j}, B_{0}\right]$.) This contradicts the previous lemma, and so $X \neq[A, B]$ for all $A, B \in R$.

We can also extend the above result to a more general setting.

Proposition 20. Suppose $F$ is a field, $R$ a commutative $F$-algebra, $I \subseteq R$ an ideal such that $R / I=F$, and $I / I^{2}$ is at least 3-dimensional over $F$. Then there exists a matrix $X \in \mathrm{M}_{2}(R)$ that has trace 0 but is not a commutator.

Proof: Let $x, y, z \in I$ be such that $\{\bar{x}, \bar{y}, \bar{z}\}$ is $F$-linearly independent in $I / I^{2}$. Then, there is a homomorphism $\phi: R \rightarrow F\left[x_{11}, x_{12}, x_{21}\right] /\left(x_{11}, x_{12}, x_{21}\right)^{2}$ such that $\phi(x)$ 
$=\bar{x}_{11}, \phi(y)=\bar{x}_{12}$, and $\phi(z)=\bar{x}_{21}$. The matrix

$$
X=\left(\begin{array}{rr}
x & y \\
z & -x
\end{array}\right)
$$

will then have the desired properties, since its image in $\mathbf{M}_{2}\left(F\left[x_{11}, x_{12}, x_{21}\right] /\left(x_{11}, x_{12}, x_{21}\right)^{2}\right)$ (under the extension of $\phi$ to a matrix ring homomorphism) is not a commutator, by the previous proposition.

\section{REFERENCES}

[1] A.A. Albert and B. Muckenhoupt, 'On matrices of trace zero', Michigan Math. J. 4 (1957), 1-3.

[2] S.A. Amitsur and L.H. Rowen, 'Elements of reduced trace 0', Israel J. Math. 87 (1994), 161-179.

[3] J. Dixmier, 'Sur les algèbres de Weyl', Bull. Soc. Math. France 96 (1968), 209-242.

[4] B. Harris, 'Commutators in division rings', Proc. Amer. Math. Soc. 9 (1958), 628-630.

[5] I. Kaplansky, "Problems in the theory of rings" revisited', Amer. Math. Monthly 77 (1970), 445-454.

[6] E. Lazerson, 'Onto inner derivations in division rings', Bull. Amer. Math. Soc. 67 (1961), 356-358.

[7] P. Revoy, 'Algèbres de Weyl en caractéistique p', C. R. Acad. Sci. Paris Sr. A-B 276 (1973), A225-A228.

[8] M. Rosset, Elements of trace zero and commutators, (Ph.D. Thesis) (Bar-Ilan University, 1997).

[9] M. Rosset and S. Rosset, 'Elements of trace zero in central simple algebras', in Rings, Extensions and Cohomology, (A. Magid, Editor) (Marcel Dekker, 1994), pp. 205-215.

[10] M. Rosset and S. Rosset, 'Elements of trace zero that are not commutators', Comm. Algebra 28 (2000), 3059-3072.

[11] K. Shoda, 'Einige Sätze über Matrizen', Japan J. Math. 13 (1936), 361-365.

Department of Mathematics

University of California

Berkeley, CA 94720

United States of America

e-mail: zak@math.berkeley.edu 